\title{
LINEAR LIE CENTRALIZERS OF THE ALGEBRA OF STRICTLY BLOCK UPPER TRIANGULAR MATRICES
}

\section{PRAKASH GHIMIRE}

Abstract. Let $\mathscr{N}$ be the algebra of all $n \times n$ strictly block upper triangular matrices over a field $\mathbb{F}$. In this paper, we describe all linear Lie centralizers of $\mathscr{N}$. We also show that every linear Lie centralizer of $\mathscr{N}$ is a centralizer. Mathematics subject classification (2010): Primary 16W25; Secondary 15B99, 15A78, 47L35, 47B47. Keywords and phrases: Centralizer, Lie centralizer, strictly block upper triangular matrix.

\section{REFERENCES}

[1] Driss Aiat Hadj And Hadj Ahmed, Non-additive Lie centralizer of strictly upper triangular matrices, Extracta Mathematics, pages 77-83, 02, 2019.

[2] JORDAN BOUNDS, Commuting maps over the ring of strictly upper triangular matrices, Linear Algebra and its Applications, 507: 132-136, 2016.

[3] M. BRes AR Centralizing Mappings and Derivations in Prime Rings, Journal of Algebra, 156 (2): 385-394, 1993.

[4] AJdA FoŠNER AND WU JING, Lie centralizers on triangular rings and nest algebras, Advances in Operator Theory, 4, 07, 2018.

[5] Prakash Ghimire and Huajun Huang, Derivations of the Lie algebra of strictly block upper triangular matrices, 2016.

[6] Prakash Ghimire and Huajun Huang, Lie Triple Derivations of the Lie Algebra of Dominant Block Upper Triangular Matrices, Algebra Colloquium, 25 (03): 475-492, 2018.

[7] J. Ghomanjani And M. A. Bahmani, A note on Lie centralizer maps, Palest. J. Math., 7 (2): 468-471, 2018.

[8] G. HochsChILD, Cohomology and representations of associative algebras, Duke Math. J., 14 (4): 921-948, 12, 1947.

[9] B. E. Johnson, Centralisers on Certain Topological Algebras, Journal of the London Mathematical Society, s1-39 (1): 603-614, 1964.

[10] B. E. Johnson, An Introduction to the Theory of Centralizers, Proceedings of the London Mathematical Society, s3-14 (2): 299-320, 1964.

[11] Shikun Ou, Dengyin WANG AND Ruiping Yao, Derivations of the Lie algebra of strictly upper triangular matrices over a commutative ring, Linear Algebra Appl., 424 (2-3): 378-383, 2007.

[12] Joso Vukman, An identity related to centralizers in semiprime rings, Commentationes Mathematicae Universitatis Carolinae, 40 (3): 447-456, 1999.

[13] Joso VUKMAN, Identities related to derivations and centralizers on standard operator algebras, Taiwanese Journal of Mathematics, 11 (1): 255-265, 2007.

[14] Joso Vukman and Irena Kosi-Ulbl, On centralizers of semiprime rings, Aequationes Mathematicae, 66 (3): 277-283, Dec 2003.

[15] Heng-TaI Wang AND QING-GuO Li, Lie triple derivation of the Lie algebra of strictly upper triangular matrix over a commutative ring, Linear Algebra Appl., 430 (1): 66-77, 2009.

[16] Qiong Wei And Pengtong Li, Centralizers of J-subspace lattice algebras, Linear Algebra and its Applications, 426 (1): 228-237, 2007.

[17] Borut Zalar, On centralizers of semiprime rings, Commentationes Mathematicae Universitatis Carolinae, 32 (4): 609-614, 1991 